FORMATION Formation emploi

Revue française de sciences sociales

113 | janvier-mars 2011

La flexicurité à l'aune de l'approche par les capacités

\title{
Déchiffrer deux indicateurs européens de flexicurité à l'aune de l'approche par les capacités
}

Decoding two European flexicurity indicators using the capability approach

Entschlüsselung zweier europäische Flexicurity-Indikatoren nach dem Maßstab

des Capability Approach

Descifrar dos indicadores europeos de flexiguridad según el enfoque de

capacidades

Jean-Michel Bonvin, Éric Moachon et Josiane Vero

\section{OpenEdition}

Journals

Édition électronique

URL : http://journals.openedition.org/formationemploi/3215

DOI : 10.4000/formationemploi.3215

ISSN : 2107-0946

Éditeur

La Documentation française

Édition imprimée

Date de publication : 1 mars 2011

Pagination : 15-32

ISSN : 0759-6340

Référence électronique

Jean-Michel Bonvin, Éric Moachon et Josiane Vero, « Déchiffrer deux indicateurs européens de

flexicurité à l'aune de l'approche par les capacités », Formation emploi [En ligne], 113 | janvier-mars

2011, mis en ligne le 10 avril 2013, consulté le 30 octobre 2020. URL : http://journals.openedition.org/ formationemploi/3215; DOI : https://doi.org/10.4000/formationemploi.3215 


\section{DOSSIER}

\section{Déchiffrer deux indicateurs européens de flexicurité à l'aune de l'approche par les capacités}

par Jean-Michel Bonvin, Éric Moachon et Josiane Vero*

Les directives européennes visent le développement de l'employabilité, conçue comme adaptabilité accrue aux exigences du marché du travail. En contrepoint,

l'approche par les capacités suggère une autre conception de la flexicurité et donc d'autres instruments pour la mesurer.

En 2007, l'Union européenne (UE) formalise l'idée d'un compromis entre flexibilité et sécurité dans une communication intitulée "Vers des principes communs de flexicurité : Des emplois plus nombreux et de meilleure qualité en combinant flexibilité et sécurité ». La volonté de la Commission européenne d'instaurer la « flexicurité » comme socle commun du marché du travail européen appelle les États-Membres à mettre en œuvre « une stratégie intégrée visant à améliorer simultanément la flexibilité et la sécurité sur le marché du travail » (Commission européenne, 2007). La formation tout au long de la vie y intervient comme une des quatre composantes aux côtés de «la souplesse et la sécurisation des dispositions contractuelles", mais aussi de "politiques actives $d u$ marché du travail » et de «systèmes de sécurité sociale modernes ».
* Jean-Michel Bonvin est professeur de sociologie et de politiques sociales à la Haute école de travail social et de la santé, EESP, Lausanne. Ses champs de compétence incluent l'étude des politiques du marché du travail dans une perspective comparative, la sociologie des entreprises et de l'administration publique, les théories de la justice économique et sociale. II a notamment publié : Amartya Sen, une politique de la liberté, Michalon, 2008 (avec Nicolas Farvaque) et "Une flexicurité au service des capacités des citoyens européens", Revue de I'IRES n 63, 2009/4 lavec Pascale Vielle).

Éric Moachon est sociologue et chercheur à la Haute école de travail social et de la santé, EESP, Lausanne. Ses travaux portent sur les instruments de gestion des politiques publiques, en particulier dans le champ des 
politiques actives du marché du travail. II a récemment publié : "What does activation mean in practice? The case of active labour market policies in Switzerland », in Vladimirova Katia (ed) Transformations in Labour: Social Security and Social Dialogue, Ciela, 2010 et "Les dilemmes des conseillers des agences publiques pour l'emploi », SociologieS, Dossiers, 2010 lavec Jean-Michel Bonvin)

Josiane Vero est économiste, ingénieur de recherche au Céreq et y a coordonné le premier dispositif d'enquêtes couplées Employeurs-salariés sur la formation, DIFES 1. Ses travaux portent sur les politiques et les pratiques de formation continue en entreprise, les politiques du marché du travail, les théories de la justice économique et sociale. Elle a récemment publié : "Trayectorias laborales y enfoque de las capacidades. Elementos para una evaluación longitudinal de las políticas de protección social", Sociología del Trabajo, 2009, n 167, pp. 127-150, lavec Joan Miquel Verd et Martí López Andreu) », et "Aspirer à se former : la responsabilité des entreprises en question », Bref, 2010, n²79 (avec Marion Lambert).

Depuis cette communication, la question de l'équilibre entre flexibilité et sécurité est devenue l'une des priorités de l'agenda européen. Cet agenda est mis en œuvre par la méthode ouverte de coordination (MOC). La MOC repose sur le principe de subsidiarité pour le déploiement de politiques nationales, déléguant à chaque État-Membre le soin de déterminer la manière dont il mettra en œuvre le modèle de flexicurité. Substitutive à la méthode communautaire classique, qui se décline sur le mode des directives «top-down » (descendantes), la MOC s'en différencie nettement. Ici, l'UE n'édicte pas de lois ou de textes contraignants, mais des lignes directrices assorties de calendriers spécifiques pour la réalisation d'objectifs généraux. Les États-Membres les traduisent en politiques nationales ou régionales. Ils rédigent ensuite des plans d'action indiquant comment ils envisagent de mettre en œuvre ces principes. La Commission analyse ces documents, rédige avec le Conseil des Ministres un rapport conjoint qui évalue les actions des États-Membres à l'aune d'un jeu d'indicateurs de performance élaborés par le «groupe sur les indicateurs » du Comité de l'emploi (EMCO) et émet un certain nombre de recommandations. À la lumière de cet exercice, les directives peuvent être révisées en vue de l'exercice suivant (Raveaud, 2006 ; Bonvin \& Vielle, 2009 ; Gazier \& Lechevalier, 2006).

En la matière, l'Union européenne n'en est pas à ses débuts. Dès le lancement de la stratégie européenne pour l'emploi, en 1997, l'usage d'indicateurs de performance, selon la méthode dite de benchmarking, (étalonnage des performances), s'est imposé comme outil de pilotage pour suivre la réalisation des objectifs établis au niveau supranational, suscitant alors une crainte d'instrumentalisation des politiques du marché du travail dans le but d'optimiser des indicateurs quantitatifs (Salais, 2004). On s'accorde d'ailleurs largement sur la nécessité de voir dans les indicateurs de performance autre chose qu'un outil neutre et indiscutable (Perret, 2002 ; Stigtlitz, Sen \& Fitoussi, 2009 ; Gadrey \& Jany-Catrice, 2005 ; Desrosrières, 2008 ; Méda 2008). Une particularité commune à ces nombreux débats est de reconnaître l'intrication des questions normatives ou politiques et des questions statistiques. Les indicateurs occupent ainsi une place de choix qu'il convient de questionner.

Dès lors, notre article vise à montrer que la construction d'indicateurs emprunte des chemins aussi scientifiques qu'idéologiques. À cet effet, il cherchera à clarifier les fondements normatifs des repères statistiques. Il montrera que de tels indicateurs, inclus dans la boîte à outil des évaluateurs, ne sauraient être envisagés comme le signe de l'alignement inéluctable sur une conception unique de la flexicurité. Dans l'ensemble, la base informationnelle mobilisée par les indicateurs se caractérise par son hétérogénéité et par une absence de fondement théorique clair. Elle résulte d'un compromis entre plusieurs inspirations possibles, ce qui n'empêche pas qu'elle cautionne un rapport déséquilibré entre flexibilité et sécurité.

Se basant sur les concepts de capacités et de base informationnelle de justice, cet article discutera les limites de la perspective à l'œuvre qui privilégie flexibilité plutôt que liberté réelle des travailleurs, employabilité plutôt que capacité, et capital humain plutôt que développement professionnel. L'alternative proposée repose sur l'approche par les capacités d'Amartya Sen. Elle conduit à privilégier l'évaluation du niveau de développement d'une société à l'aune de la liberté réelle dont ses membres disposent pour choisir leur mode d'existence. Une des spécificités de l'approche par les capacités est de conjuguer une perspective 
d'évaluation de la liberté d'agir à une perspective normative qui fait de l'égale distribution de cette liberté un principe de justice (Sen, 2010). Ainsi, l'exercice de la responsabilité exige une latitude de choix entre différentes options possibles et un pouvoir de convertir l'option choisie en réalisation effective.

Cette contribution est organisée en trois temps. D'abord, nous reviendrons sur le contexte dans lequel l'Europe s'est dotée d'indicateurs de flexicurité, avant de les présenter. L'attention portera sur les deux indicateurs dynamiques de synthèse que sont d'une part l'indicateur de performance dans la sécurité de l'emploi et d'autre part celui de progrès des qualifications. Attestant de la fonction éminemment politique des indicateurs dans la gouvernance de la flexicurité, la deuxième partie s'attachera à révéler les normes et les valeurs qui président à leur construction. En contrepoint, nous développerons l'approche par les capacités $(\mathrm{AC})$ comme manière alternative de concevoir la sécurité et le rôle qu'est susceptible d'y jouer la formation. Pour conclure, nous poserons quelques repères en vue de la mise en œuvre, à l'échelon européen, de bases de données susceptibles d'améliorer la connaissance vers la liberté réelle de travailler et de se développer professionnellement au travers de la formation.

\section{FLEXICURITÉ : DES PRINCIPES AUX INDICATEURS}

À l'origine du projet de flexicurité en Europe se trouve un constat largement partagé dès le milieu des années 90 : l'ampleur, liée à la mondialisation, des flux de destruction et de création d'emploi, l'intensification de la concurrence et le développement massif des nouvelles technologies. Selon ce constat, la sécurité de l'emploi, ébranlée par les exigences de la production flexible, ne peut plus suffire. Il importe donc de sécuriser autant que possible les parcours professionnels, en imaginant des moyens de garantir la sécurité des travailleurs européens. Le modèle de flexicurité, qui se situe au cœur des débats en Europe, vise à proposer une stratégie de la sécurité par l'employabilité, dont l'ambition est de faciliter la prise d'un nouvel emploi au sein de la même entreprise ou chez un autre employeur.

\section{La flexicurité s'impose comme un concept clef de la stratégie européenne pour l'emploi}

En prétendant concilier deux réalités considérées a priori comme antinomiques, la flexicurité s'est imposée comme un concept politique essentiel de la stratégie européenne pour l'emploi (SEE). Ce concept est alors transposé en recommandation dans le cadre des lignes directrices pour l'emploi. Ainsi, en 2005, la ligne 21 vise à « favoriser la flexibilité en la conciliant avec la sécurité de l'emploi et à réduire la segmentation du marché du travail, en tenant dûment compte du rôle des partenaires sociaux $»^{1}$. Un an plus tard, le Rapport sur l'emploi en Europe appelle les États-Membres à une convergence sur ces principes communs (Commission européenne, 2006a). Dans le même temps, la Commission publie un livre vert fournissant quelques principes directeurs pour les 27 pays membres, qu'elle livre au débat public (Commission européenne, 2006b). Enfin, une communication de la Commission formalise les voies concrètes de la flexicurité à partir de 2007 (Commission européenne, 2007).

Le succès de certaines expériences nationales, comme celles du Danemark ou des Pays-Bas, a largement inspiré la pensée européenne, dans un contexte de fragilisation du modèle fordiste combinant compétitivité économique et sécurité sociale. Dès le milieu des années 90, de nombreux analystes soulignent l'ébranlement de la sécurité de l'emploi qui constituait, jusque-là, un repère pour la conduite et l'évaluation des politiques économiques en Europe : le rapport Supiot (1999), qui annonce la pluralisation des normes d'emploi et la remise en cause de la dualisation du marché du travail face à l'inéluctable flexibilité, les partisans des marchés transitionnels du travail qui envisagent la discontinuité des parcours professionnels comme un fait acquis (Wilthagen, 1998 ; Schmid et Gazier, 2002), le courant néo-institutionnaliste visant à identifier les divers régimes d'État-providence à la suite d'EspingAndersen $(1999,2007)$, ou encore les travaux qualifiant les formes contemporaines de capitalisme (Hall et Soskice, 2001). Un consensus émerge quant à la nécessité de flexibiliser le marché du travail et de repenser la sécurité dans ce nouveau contexte. Le passage

\footnotetext{
${ }^{1}$ Lignes directrices pour l'emploi 2005-2008, JO, 6.08.2005.
} 
progressif d'une logique de sécurité de l'emploi à celle d'une sécurisation active des parcours professionnels devient désormais le défi à relever.

Pour autant, la Commission n'envisage pas une voie unique de la flexicurité. Elle se réfère en cela aux travaux de Wilthagen qui présentent la flexicurité comme le résultat de combinaisons différentes de diverses dimensions de la flexibilité et de la sécurité. Elle peut croiser des éléments de flexibilité interne, externe, fonctionnelle et salariale avec des formes associées de sécurité : sécurité de l'emploi, sécurité d'employabilité, sécurité du revenu ou «sécurité combinée », par exemple l'équilibre entre la vie professionnelle et familiale (Wilthagen \& al. 2003, Wilthagen \& Tros, 2004). Ainsi, toutes les politiques qui tentent explicitement de trouver un équilibre entre la flexibilité et la sécurité ont été rassemblées sous l'étiquette commune de la stratégie de flexicurité. La Commission européenne la définit comme «stratégie intégrée visant à améliorer simultanément la flexibilité et la sécurité sur le marché du travail. En d'autres termes, la flexicurité est une approche globale du marché du travail qui allie flexibilité des modalités contractuelles, sécurité dans l'emploi (garantie de rester à son poste ou de trouver un autre travail rapidement) et gestion adaptée des périodes de transition » (Commission européenne, 2007).

Formulé en termes généraux, ce modèle emporte l'adhésion : face à la globalisation des échanges économiques et financiers, la compétitivité implique plus de flexibilité dans les processus de production. Il importe donc d'amortir les conséquences d'une telle flexibilité au travers de démarches de sécurisation des droits et des parcours professionnels. Ici, la rhétorique de la flexicurité repose sur l'hypothèse que la flexibilité et la sécurité ne sont pas contradictoires. On occulte toutes les tensions normatives pourtant inhérentes à ce concept. La réconciliation des objectifs de flexibilité et de sécurité ne va pas de soi. Il est alors facile d'imaginer que les visions des partenaires en présence divergeront de manière substantielle lorsqu'il s'agira de traduire la flexicurité dans les faits ; et ce d'autant que les principes communs de la Commission sont sibyllins quant aux réformes concrètes attendues, depuis que la Méthode ouverte de coordination (MOC) est devenue le modèle pour résoudre les problèmes complexes de gouvernance multi-niveaux.

\section{L'Europe se dote de tableaux de bord pour gouverner la flexicurité}

Prônée dans le cadre de la flexicurité, la Méthode ouverte de coordination (MOC) a été formulée au sommet de Lisbonne de mars 2000, en réaction à la méthode communautaire, jugée trop contraignante (Zeitlin \& Pochet, 2005). Elle s'affiche néanmoins comme un dispositif visant à renforcer la « dimension communautaire ». Les efforts de tous les ÉtatsMembres doivent en effet converger pour atteindre des objectifs définis à l'échelon européen dans des domaines qui relèvent en grande partie de leur compétence. À l'encontre de la méthode communautaire, la MOC se caractérise par une forme de souplesse, qui laisse aux États-Membres le choix de mettre en œuvre, de manière volontaire et réversible, des actions dans des domaines de compétences qui, sur le principe, restent nationaux. Pour autant, cela ne signifie pas qu'il y ait une liberté totale. La MOC implique un processus de monitoring (système de suivi de la mise en œuvre d'un processus, d'une politique) par étapes, où, à l'origine, le Conseil des ministres de l'UE fixe les objectifs politiques. Puis, les États-Membres traduisent ces lignes directrices en politiques nationales et/ou régionales. On s'entend ensuite sur une liste d'indicateurs spécifiques pour évaluer ces politiques et identifier les meilleures pratiques. Ce faisant, la MOC entraîne une obligation de rendre des comptes sur les résultats.

Ce nouvel art de l'efficience politique s'inspire de la nouvelle gestion publique (NGP) pour développer une régulation par objectifs. Cette perspective cherche à transposer la logique et la gouvernance managériales du secteur privé dans le domaine public, en introduisant des méthodes et outils de gestion issus des grandes entreprises privées. Ainsi sont instaurés de nouveaux modes de pilotage ou de régulation basés sur un partage des rôles entre le pouvoir politique, qui prend les décisions stratégiques et fixe les objectifs, et des structures autonomes ou renforcées dans leurs prérogatives opérationnelles à qui sont déléguées les décisions de mise en œuvre et la responsabilité de l'atteinte d'objectifs. Comme Bonvin \& Conter (2006, p. 6) l'ont souligné, elle « comporte la promesse d'un passage de la bureaucratie technocratique dans laquelle les décisions sont prises et imposées suivant 
un mode hiérarchique à des modes de gouvernance plus flexibles et situés (Salais, Storper, 1993) qui se veulent en phase avec une conception réflexive et révisable de l'action publique dans la ligne de la régulation conjointe de Reynaud (1989)».

Ainsi, l'action publique est pilotée par les indicateurs de performance et l'usage du benchmarking. Il s'agit de choisir des indicateurs de performance quantifiables, de recueillir de manière centralisée les statistiques nationales relatives à ces indicateurs, de mesurer les écarts entre les résultats enregistrés en vue de comparer les politiques gouvernementales. Au même titre que le dirigeant d'une entreprise, l'Union européenne établit un tableau de bord composé d'indicateurs quantitatifs à atteindre. Chacune des performances d'un État-Membre est comparée annuellement aux autres; et chacun des résultats, à celui de l'exercice précédent. L'indicateur définit le champ pratique et les critères qui présideront au benchmarking, c'est-à-dire à l'étalonnage de la performance des États-Membres.

L'introduction de logiques issues du secteur privé ne peut toutefois pas être considérée comme allant de soi. Plus que la question de son inspiration ou des modalités de sa mise en œuvre, ce qui importe ce sont ses effets sur le comportement des États-membres. Comme Salais $(2004,2010)$ l'a écrit avant nous, le pilotage de l'action publique par les indicateurs de performance peut conduire à une dérive instrumentaliste. Plutôt que de viser une connaissance objective des faits afin de mener des politiques réellement adéquates, le risque est que l'action publique s'organise autour du processus de production du chiffre attendu. «Il s'agirait à terme d'une inversion/perversion des rapports entre connaissance et décision publique, ainsi que de la décision publique elle-même. La dérive qui se produit est simple dans son principe. Disposant à l'avance de ses cibles quantitatives, lesquelles sont en même temps les critères par lesquels s'évalue la performance qui sera publiée et affichée, la décision publique et sa mise en ouvre se fixent peu à peu comme objectif d'améliorer directement leur score, tel que mesuré par les indicateurs. L'enjeu n'est plus d'améliorer réellement les situations (ce réel étant constaté par des méthodes d'enquête et de recueil de données qui privilégient une connaissance véritable des situations de vie et de travail), mais d'obtenir la performance quantitative souhaitée, quelle qu'en soit la méthode. » (Salais, 2010, p. 135). Les États-Membres sachant ce qu'ils doivent accomplir pour être bien notés, il est à craindre que leurs choix politiques et les programmes qu'ils mettront en œuvre se calqueront autant que possible sur les indicateurs de performance définis. Dès lors, ces derniers apparaissent comme un révélateur des enjeux de la flexicurité. C'est en effet plus à leur niveau qu'à celui des grands principes contenus dans la stratégie européenne que se manifestent avec le plus de clarté ces enjeux.

\section{L'Europe crée deux indicateurs dynamiques}

À partir de 2006, le « groupe sur les indicateurs »du Comité de l'emploi engage ses travaux. Ils visent à formaliser les quatre composantes de la flexicurité : assouplissement de la législation sur la protection de l'emploi, formation tout au long de la vie, politiques actives du marché du travail et modernisation du système de protection sociale. Pour chacune d'entre elles, le groupe s'est doté d'un jeu d'indicateurs censés refléter le niveau de flexicurité atteint par chaque pays, en s'appuyant sur les indicateurs déjà élaborés dans le cadre de la SEE ou explorant les indicateurs disponibles en provenance de l'OCDE Organisation de coopération et de développement économiques (cf. rapport Monitoring and Analysis of Flexicurity Policies, Commission européenne, 2009, juillet 2009) (Encadré 1).

Afin de décrire la sécurité du parcours ou des périodes de transition professionnelles, le « groupe sur les indicateurs » a développé des indicateurs dynamiques sur le processus de transition, plus adaptés que les indicateurs d'état à un moment donné. Dans cette quête, il a privilégié des indicateurs de synthèse afin d'obtenir une vision simple et claire de la situation de chaque État-Membre. Faute de sources comparables, le groupe n'a pas réussi à construire des indicateurs pour chacune des quatre dimensions. Cela explique que l'on ne dispose pas d'indicateurs dynamiques pour les "politiques actives du marché du travail » ni pour les «systèmes de sécurité sociale modernes». Néanmoins, la disponibilité de données longitudinales issues du nouveau panel européen SILC (Survey on Income and Living Conditions), a permis de se doter 


\section{Encadré 1 \\ Indicateurs européens de flexicurité (juillet 2009)}

Indicateurs de la SEE pour piloter l'assouplissement de la législation sur la protection de l'emploi

\begin{tabular}{|c|l|l|}
\hline \multicolumn{1}{|c|}{ Indicateurs d'Input } & \multicolumn{1}{|c|}{ Indicateurs de processus } & \multicolumn{1}{c|}{ Indicateur d'output } \\
\hline - Accès à des horaires flexibles & $\begin{array}{l}\text { - Part des travailleurs avec des } \\
\text { contrats atypiques (CDD, temps } \\
\text { partiel) } \\
\text { - Travailleurs réalisant des heures } \\
\text { supplémentaires }\end{array}$ & $\begin{array}{l}\text { - Transitions par type de contrat } \\
\text { - Heures supplémentaires }\end{array}$ \\
\hline
\end{tabular}

Indicateurs de la SEE pour piloter la Formation tout au long de la vie

\begin{tabular}{|c|c|c|}
\hline Indicateurs d'Input & Indicateurs de processus & Indicateur d'output \\
\hline $\begin{array}{l}\text { - Dépenses publiques d'éducation } \\
\text { - Investissement des entreprises } \\
\text { dans la formation des adultes }\end{array}$ & $\begin{array}{l}\text { - Taux de participation de la popu- } \\
\text { lation à la formation continue lâge } \\
25-64 \text { ) : } \\
\text { - Taux de participation des sala- } \\
\text { riés à la formation professionnelle } \\
\text { continue }\end{array}$ & $\begin{array}{l}\text { - Transitions entre statut d'emploi et } \\
\text { niveau de rémunération } \\
\text { - Niveau d'éducation des adultes } \\
\text { - Compétences des adultes en } \\
\text { usage de l'informatique }\end{array}$ \\
\hline
\end{tabular}

Indicateurs de la SEE pour piloter les Politiques actives du marché du travail (PAMT)

\begin{tabular}{|l|l|l|}
\hline \multicolumn{1}{|c|}{ Indicateurs d'Input } & \multicolumn{1}{|c|}{ Indicateurs de processus } & \multicolumn{1}{c|}{ Indicateur d'output } \\
\hline - Dépenses en PAMT par deman- & $\begin{array}{l}\text { • Taux d'activation des demandeurs } \\
\text { d'emploi } \\
\text { deur d'emploi ; }\end{array}$ & $\begin{array}{l}\text { - Taux de retour à l'emploi des } \\
\text { participants aux PAMT }\end{array}$ \\
& $\begin{array}{l}\text { Teunes chômeurs et des chômeurs } \\
\text { adultes }\end{array}$ & \\
\hline
\end{tabular}

Indicateurs de la SEE pour piloter la modernisation du système de protection sociale

\begin{tabular}{|c|c|c|}
\hline Indicateurs d'Input & Indicateurs de processus & Indicateur d'output \\
\hline $\begin{array}{l}\text { - Dépense d'indemnisation par } \\
\text { demandeur d'emploi } \\
\text { - Dépense d'indemnisation du } \\
\text { chômage en \% du PIB } \\
\text { - Trappe à chômage } \\
\text { - Trappe à bas salaires } \\
\text { - Taux de garde d'enfants de moins } \\
\text { de trois ans } \\
\text { - Taux de prise en charge de } \\
\text { personnes âgées dépendantes }\end{array}$ & $\begin{array}{l}\text { - Taux d'indemnisation des deman- } \\
\text { deurs d'emploi } \\
\text { - Taux d'emploi des personnes } \\
\text { ayant des enfants }\end{array}$ & $\begin{array}{l}\text { - Taux de pauvreté des DE } \\
\text { - Manque de garde d'enfants ou } \\
\text { de personnes âgées dépendantes } \\
\text { - Baisse des taux de remplacement } \\
\text { due à des interruptions de carrière }\end{array}$ \\
\hline
\end{tabular}

Source : EMCO Report (2009), Monitoring and analysis Flexicurity policies, Issue 2, July.

Sigles : CDD : contrat à durée déterminée ; BIT : Bureau international du travail ; PAMT : Politiques actives du marché du travail ; DE : Demandeurs d'emploi ; PIB : produit intérieur brut. 
Tableau 1

Évolution de la sécurité de l'employabilité : matrice des transitions annuelles

\begin{tabular}{|c|c|c|c|c|c|c|c|}
\hline Statut en $t+1$ & $\begin{array}{c}\text { Contrat } \\
\text { permanent }\end{array}$ & $\begin{array}{l}\text { Contrat } \\
\text { temporaire }\end{array}$ & Indépendant & Chômage & Études & Retraite & $\begin{array}{c}\text { Autre } \\
\text { inactivité }\end{array}$ \\
\hline Contrat permanent & $=$ & - & - & - & - & - & - \\
\hline Contrat temporaire & + & $=$ & $=$ & - & - & - & - \\
\hline Indépendant & $+\mathrm{OU}=$ & $=$ & $=$ & - & - & - & - \\
\hline Chômage & + & + & $+*$ & - & + & - & - \\
\hline Formation - Études & + & + & + & - & $=$ & - & - \\
\hline Retraite & + & + & + & + & + & $=$ & $=$ \\
\hline Autre inactivité & + & + & + & + & + & - & $=$ \\
\hline
\end{tabular}

Source : Employment Committee (2009).

* Note de lecture : Une personne au chômage en t qui se retrouve indépendante en $\mathrm{t}+1$ se trouve dans une situation meilleure selon les concepteurs de l'indicateur de sécurité ; cette transition est donc notée au moyen du signe «+».

d'indicateurs pour les deux autres composantes de la flexicurité.

La première de ces composantes est la souplesse et la sécurisation des dispositions contractuelles. Elle recouvre des domaines allant des conditions relatives aux contrats de travail à la gestion des horaires de travail. L'indicateur dynamique, qui se situe au cœur du tableau de bord du pilotage des résultats en Europe, se fonde sur les transitions annuelles sur le marché du travail et l'évolution de l'utilisation des différents contrats. Dans ce cadre, les transitions entre deux années sont évaluées comme positives, neutres ou négatives au regard de l'évolution de la situation des personnes.

Prenant appui sur les travaux de Muffels \& Luijikx (2008), l'indicateur de sécurité d'emploi identifie et dénombre le taux de personnes ayant au moins le même niveau de sécurité d'emploi que l'année antérieure (c'est-à-dire un niveau identique ou supérieur), à partir de la matrice présentée dans le tableau 1. En résumé, pour les personnes sans emploi, la sécurité est renforcée dès lors qu'on observe un rapprochement vers le marché du travail, tandis que pour les personnes en emploi, la sécurité augmente avec celle du type de contrat de travail.

La seconde composante est la Formation tout au long de la vie (FTLV), devenue une priorité politique européenne depuis le conseil de Lisbonne, en mars 2000. Jamais les attentes à son égard n'ont paru aussi fortes. La protection des salariés y intervient au premier plan, à côté de trois autres objectifs : procurer aux entreprises les rendements d'un investissement productif, favoriser la croissance de l'emploi, et enfin constituer, pour la collectivité, un facteur de compétitivité globale.

L'indicateur de progrès de qualification vise à fournir un score sur les effets des investissements en formation, en mesurant la proportion de personnes ayant au moins le même niveau de qualification que l'année précédente. Les transitions entre deux années sont évaluées comme positives, neutres ou négatives au regard d'un double critère : la mobilité sur le marché du travail (formation, emploi, inactivité et chômage) et la mobilité salariale. En résumé, pour les personnes sans emploi, la qualification est renforcée dès lors qu'on se rapproche du marché du travail ou d'une situation de formation, tandis que pour les personnes en emploi ou en formation, la qualification s'accroît avec une mobilité salariale ascendante, comme en témoigne la matrice présentée dans le tableau 2.

En incitant les États-Membres à améliorer leur rang dans le palmarès résultant des indicateurs chiffrés, les indicateurs prescrivent des priorités. Ainsi, ce qui sera mesuré au moyen d'indicateurs de performance, c'est aussi ce qui sera réalisé. Ce qui n'est pas mesuré, c'est donc aussi ce qui sera probablement omis. Ces indicateurs se présentent donc comme un révélateur des enjeux de la flexicurité que nous allons à présent examiner. 
Tableau 2

Évolution de la qualification : matrice des transitions annuelles

\begin{tabular}{|l|c|c|c|c|c|c|}
\hline & $t+1$ & $\begin{array}{c}\text { Formation } \\
\text { Études }\end{array}$ & $\begin{array}{c}\text { Autre } \\
\text { inactivité }\end{array}$ & Chômage & $\begin{array}{c}\text { Emploi } \\
\text { Décile 1 } \\
\text { (salaire) }\end{array}$ & $\begin{array}{c}\text { Emploi } \\
\text { Décile 10 } \\
\text { (salaire) }\end{array}$ \\
\hline Formation & + & - & - & + & + & + \\
\hline Autre inactivité & + & $=$ & + & + & + & + \\
\hline Chômage & + & - & $=$ & + & + & + \\
\hline Emploi - Décile 1 (salaire) & + & - & - & $=$ & + & + \\
\hline$\ldots$ & + & - & - & - & + & + \\
\hline Emploi - Décile 10 (salaire) & + & - & - & - & - & $=$ \\
\hline
\end{tabular}

Source : Employment Committee (2009).

* Note de lecture : Une personne au chômage en t qui se retrouve en emploi en $\mathrm{t}+1$ avec une rémunération comprise dans le premier décile des salaires (le premier décile correspond aux $10 \%$ de salariés les moins bien payés) se trouve dans une situation meilleure selon les concepteurs de l'indicateur de qualification; cette transition est donc notée au moyen du signe «+».

\section{DES INDICATEURS POUR MESURER QUOI ?}

Les indicateurs constituent des outils à double tranchant. Au titre des bénéfices attendus est souvent citée leur capacité à rendre visibles et compréhensibles des situations complexes. Parmi les effets pervers évoqués surgit la crainte d'occulter les « conventions de la quantification ». Ainsi, la gouvernance par les indicateurs repose sur l'hypothèse que les catégories statistiques représentent adéquatement les situations et sur l'oubli des conventions et des méthodes qui ont présidé à la construction de ces catégories (Salais, 2004 ; Supiot, 2010). ${ }^{2}$

Les travaux conduits par Sen permettent d'appréhender la trame normative des conventions de la quantification, notamment grâce à l'idée centrale de « base informationnelle» de jugement. Celle-ci «identifie l'information sur laquelle repose directement le jugement et - tout aussi important-assure que la vérité ou la fausseté des autres informations ne peut directement influencer la justesse du jugement. La BIJ détermine le territoire factuel sur lequel les considérations relatives à la justice s'appliquent directement » (Sen, 1990, p. 11).

\footnotetext{
2 Alain Desrosières rappelle également que « Le gouvernement des hommes et la mise en scène de la nature par les savants ont, l'un et l'autre, beaucoup recours à l'argument statistique. La quantification, signe d'objectivité, de rigueur et d'impartialité, est mobilisée dans des situations fort variées »(Desrosières 2008, p. 7).
}

Ainsi, les indicateurs ne peuvent pas être considérés comme un reflet du monde; ils en fournissent une image partielle en sélectionnant une base informationnelle spécifique, aux dépens d'autres bases possibles. De la sorte, ils contribuent aussi à reconfigurer la situation observée dans le sens des valeurs et normes qui leur sont sous-jacentes. En effet, même lorsqu'ils reposent sur des informations objectives et irréfutables, ils épousent des jugements de valeur, souvent passés sous silence ou considérés comme allant de soi, sur la pertinence des informations à retenir, aux dépens d'autres faits considérés comme inappropriés. Sen désigne cette nécessaire partialité des indicateurs au moyen du concept d' « objectivité positionnelle » qui souligne le fait que, suivant la position qu'on occupe, on tend à privilégier un point de vue sur la réalité qu'on observe au détriment d'autres points de vue. Il n'y a donc pas d'objectivité absolue, ni dans la connaissance scientifique, ni dans le raisonnement éthique. La conception de ce qu'est une description adéquate ou un fonctionnement (une manière d'être ou de se comporter) de valeur dépend de la position ou de la situation de l'acteur. La sélection de la base informationnelle n'a pas seulement des effets descriptifs (dans la mesure où elle met l'accent sur un mode spécifique de description de la réalité), elle exerce aussi des effets de transformation de cette réalité. De fait, avec les indicateurs, l'accent est aussi mis sur les rapports entre description et prescription. Décrire des situations implique de faire des choix et d'attirer l'attention des décideurs publics et de l'opinion sur 
les questions considérées comme les plus importantes. L'élaboration d'indicateurs n'a pas seulement pour visée une description de l'existant ni une analyse des pratiques, elle est d'abord une action politique qui se conjugue à une dimension prescriptive.

Les experts du "groupe sur les indicateurs" n'échappent bien sûr pas à cette nécessité de sélectionner une base informationnelle et à la condition de l'objectivité positionnelle, qui est fondatrice de toute démarche épistémologique. Il importe donc de s'interroger sur les soubassements normatifs et informationnels des deux indicateurs dynamiques de flexicurité à la lumière des principes épistémologiques de Sen. Notre propos consiste ainsi à mettre en lumière les conventions de la quantification inhérentes aux indicateurs de flexicurité, tout en les mettant à l'épreuve de la conception alternative de la flexicurité tracée par l'approche par les capacités. Dans un premier temps, nous porterons notre attention sur l'indicateur de sécurité dans l'emploi. Nous analyserons ensuite l'indicateur d'évolution de qualification.

\section{La sécurité comme adaptabilité aux exigences du marché du travail}

Pour R. Salais, «le grand basculement qu'introduit l'approche par les capacités est relatif au choix de la référence par rapport à laquelle l'action publique (les politiques, la législation, les procédures) doit être conçue, mise en œuvre et évaluée. Pour Sen, la seule référence éthiquement légitime de l'action publique est la personne, précisément son état quant à l'étendue des libertés réelles dont elle dispose pour choisir et conduire la vie qu'elle entend mener » (Salais, 2005, p. 10). Une telle conception trace une voie ambitieuse pour les politiques publiques, qui ne se réduit pas à favoriser l'adaptabilité des personnes aux exigences du marché du travail, mais tend à promouvoir leur liberté réelle de choisir la vie qu'elles ont de bonnes raisons de mener. L'action publique est ainsi appelée à développer les opportunités à disposition des personnes tout en reconnaissant leur liberté de choix en matière de modes de vie ou de manières d'être.

Par contraste, la matrice de l'indicateur de sécurité d'emploi ( $c f$. tableau 1) montre que la définition de la transition de valeur est confisquée par les experts. La perspective retenue puise, pour une large part, dans les apports du réseau des marchés transitionnels. Les défenseurs de cette perspective se sont focalisés sur une typologie des transitions qu'une personne peut accomplir au sein du marché du travail, afin de repérer les transitions souhaitables. Or, la construction des repères d'objectivité sur la valeur des transitions réalisées sur le marché du travail ne peut être garantie par les experts du marché du travail. Il est à craindre un certain degré d'ethnocentrisme (où l'observateur se trompe sur l'universalité de son point de vue) ou de paternalisme (où le décideur se croit autorisé à imposer sa vision aux autres). Ceci peut conduire à classer comme positives des transitions relevant de comportements et d'aspirations simplement différents.

L'erreur statistique ne serait pas trop gênante si elle n'allait de pair avec des erreurs politiques. Décrire des transitions comme positives, c'est prescrire des transitions souhaitables. Ainsi, la transition vers l'emploi est toujours interprétée positivement, sans tenir compte de la qualité de l'emploi en question.

De la même façon, la dévalorisation de la (pré-) retraite et de toutes les formes d'inactivité est un postulat normatif fort de l'indicateur de sécurité de l'emploi. Ainsi, le passage d'un statut d'invalide à un statut de chômeur est interprété positivement en termes de sécurité. En effet, la recherche d'un emploi ou la disponibilité au placement (ce qui est un critère d'éligibilité dans le cadre de l'assurance-chômage) sont envisagées comme un progrès par rapport à une situation où la personne est autorisée à être inactive ou exerce une activité rémunérée par une rente ou autre prestation sociale au sein d'ateliers protégés (ce qui est fréquent dans le cadre de l'assurance-invalidité). Ce choix correspond bien à la volonté d'augmenter les taux d'emploi qui est au cœur de la stratégie européenne ; il voit dans l'emploi le fonctionnement de valeur par excellence ; et cela sans tenir compte de la qualité de l'emploi ou des circonstances particulières liées à la personne (en termes de capacité physique, psychologique ou autres à exercer un emploi, à concilier travail-famille, etc.).

L'indicateur dynamique envisage la sécurité sous l'angle de l'adaptation aux exigences du marché du travail, même si cela implique de sacrifier la qualité de vie ou de travail ou de renforcer la précarisation de l'emploi sur le marché du travail. Ainsi, la qualité du 
travail ou de la transition ne concerne que marginalement la politique de flexicurité.

De plus, les conventions de quantification proposent une vision réductrice des transitions dès lors que les matrices de transition annuelles sont susceptibles de gommer certains épisodes intermédiaires. La démarche reste ainsi aveugle à certaines transitions qui sont pourtant au cœur des phénomènes de précarité de l'emploi : passage par le chômage entre deux CDD ou CDI, passage d'un CDD long à un CDD court, perte de revenu dans le même décile. Elle occulte également la qualité des transitions dans l'emploi et le travail.

Si le thème de la qualité de l'emploi a été intégré à la stratégie européenne pour l'emploi lors du second semestre 2001 (au travers des dix indicateurs de Laeken), (cf. Commission européenne, 2001), il a progressivement disparu suite à la récession de 20012002. En découle, dès 2003, une révision progressive de la SEE, privilégiant la « quantité » de l'emploi à sa « qualité » et « recentrant les priorités sur la croissance et l'emploi ». La flexicurité, qui n'était alors qu'un des dix thèmes de la qualité de l'emploi, est devenue un thème central dans les réflexions de l'EMCO (Employment Commitee, comité de l'emploi). En introduisant dans l'analyse une dimension plus dynamique, elle a contribué à reléguer au second plan la thématique de la qualité de l'emploi et à faire accepter l'idée selon laquelle un mauvais emploi vaudrait mieux que pas d'emploi du tout. De fait, dans une perspective plus synchronique, la qualité de l'emploi, à un moment donné, apparaît comme une question centrale, alors que dans une optique dynamique, un mauvais emploi peut être justifié parce qu'il peut constituer un tremplin vers une insertion durable dans l'emploi.

Passer à une vision de la flexicurité inspirée de l'approche par les capacités supposerait un certain nombre de développements. Il conviendrait tout d'abord d'intégrer la question de la qualité de l'emploi dans une perspective à la fois synchronique et dynamique, qui renvoie à "une analyse de l'étendue des opportunités offertes par l'inclusion dans le marché du travail, en termes de travail et de vie » (Salais, 2006, p. 438). De plus, à l'encontre des fondements normatifs de la flexicurité telle que mesurée par l'indicateur de sécurité, l'approche par les capacités met l'accent sur les deux dimensions essentielles de la liberté réelle : le développement des opportunités (ou empowerment), grâce auquel les personnes acquièrent les moyens de la liberté de choix et le respect de la liberté processuelle grâce à laquelle elles restent maîtresses de leurs choix (la dimension processuelle de la liberté requiert des processus impliquant la participation active de chacun aux choix ou décisions qui le concernent). Que l'une de ces deux dimensions fasse défaut et l'objectif de développement des capacités est manqué.

Évaluée à cette aune, la flexicurité, telle que définie par l'indicateur de sécurité, apparaît doublement déficiente. Sur le versant opportunités, elle vise avant tout le développement de l'employabilité conçue comme adaptabilité accrue aux exigences du marché du travail. De la sorte, l'accès à la sécurité dans les autres aspects de la vie apparaît comme subordonné à l'exercice d'un emploi. Il est donc à craindre que les personnes dont l'employabilité est durablement réduite bénéficient d'une sécurité limitée. De plus, la manière dont les transitions sont valorisées et hiérarchisées indique une adhésion claire à la notion d'activation : tout progrès vers l'emploi ou vers une situation estimée plus proche de l'emploi (par exemple le chômage par rapport à l'invalidité) constituerait un progrès en termes de sécurité. Une telle conclusion apparaît beaucoup plus sujette à caution si l'on se réfère aux capacités : un emploi très précaire et mal payé permet-il réellement de développer les capacités d'un chômeur ou d'un invalide ? Dans certains cas sans doute, mais pas dans tous. À l'aune des capacités, cette question exigerait une réponse contrastée que ne permet pas le recours à l'indicateur de sécurité.

Sur le versant processus, considérer la reprise d'un emploi comme la condition sine qua non pour pouvoir disposer d'un certain degré de sécurité constitue une restriction de la liberté de choix des personnes concernées. Dans la mesure où elle établit une hiérarchie entre les diverses situations possibles à l'égard du marché du travail (être au chômage est mieux qu'être inactif, etc.), la stratégie de flexicurité impose aux autorités politiques nationales et régionales et aux individus qui en bénéficient de se conformer à cette hiérarchie et de l'inscrire concrètement dans leurs pratiques et comportements quotidiens. À défaut, les États-Membres se retrouveront avec de mauvais indicateurs et les bénéficiaires seront menacés de sanction ou de suspension de leurs droits. 
En envisageant l'organisation du marché du travail sous l'angle de la flexibilité, la stratégie de flexicurité subordonne l'accès à la sécurité à l'acceptation de la flexibilité du marché du travail, ce qui constitue à nouveau une restriction significative en termes de liberté de choix. Cette dernière n'est reconnue que dans la mesure où la personne se conforme à la conception normative sous-jacente à l'indicateur et s'inscrit dans une volonté d'adaptation aux exigences du marché du travail.

Au vu de ces éléments, l'indicateur de sécurité apparaît trop uniforme au regard de la diversité des situations. Cet écueil fonde la nécessité de situer et contextualiser les questions de justice, au rang desquelles figure la question des indicateurs de performance utilisés dans l'évaluation des politiques publiques. À cet égard, Amartya Sen n'établit aucun critère de justice unique et définitif. L'incomplétude de sa théorie de la justice se présente comme la condition même d'actualisation de sa vision constructive de la démocratie (Bonvin, 2005). Sous cet angle, tous les choix collectifs qui ne laissent pas d'espace à la perspective de la liberté réelle des personnes sont critiquables car ils reflètent la vision des concepteurs d'indicateurs. À l'image de l'initiative des Réseaux canadiens de recherche en politique publique (RCRPP) ${ }^{3}$, une vaste consultation de la population européenne, visant à inciter les citoyens à s'exprimer sur le choix d'indicateurs, rejoindrait les préoccupations démocratiques de l'approche par les capacités. Une démarche similaire a également été tentée en Belgique (Orianne \& al., 2010), en vue de renouveler le cadre des indicateurs dynamiques de flexicurité à partir d'un travail définitionnel participatif. Suivant Orianne et al. (2010), celui-ci consiste à réaliser, en groupe avec les acteurs du marché de l'emploi, un travail réflexif et collectif autour de la notion de transition positive.

\section{La formation conçue comme capital humain}

La formation est érigée, dans le débat européen, en levier tout à la fois de sécurisation des parcours individuels et d'édification de la société de la connaissance. Dès lors, nous souhaitons ici examiner la manière

${ }^{3}$ Sur la participation des citoyens, dans le cadre des RCRPP
cf. http://www.cprn.org/theme.cfm?l=fr\&theme $=109$. dont elle est envisagée dans l'indicateur dynamique au cœur des évaluations de la flexicurité. Deux postulats normatifs caractérisent cet indicateur.

En premier lieu, la formation est considérée comme un bien, un plus, quels que soient son contenu, sa qualité ou sa durée et quel que soit le statut de la personne concernée (pour un chômeur ou un invalide, au même titre que pour une personne en CDD ou en CDI). Or, suivre une formation qui familiarise avec les techniques les plus efficientes de la recherche d'emploi, comme la rédaction de lettres de motivation ou de CV attractifs, n'a pas la même portée que la participation à une formation qualifiante de longue haleine. Toutefois, signalons ici que la continuation de la formation d'une année à l'autre est envisagée de manière positive (c'est même la seule occurrence dans le tableau 2 où le fait de se retrouver dans une situation identique d'une année à l'autre est apprécié positivement); ce qui indique une volonté, trop sommaire cependant, de saisir la durée de la formation comme un avantage pour la personne concernée. De plus, acquérir des compétences transversales et donc transférables qui permettent d'améliorer l'employabilité des personnes sur le marché du travail n'est pas la même chose que de suivre une formation d'adaptation au poste de travail centrée sur des compétences spécifiques, reconnues dans une seule entreprise. L'indicateur ne permet pas de différencier adéquatement ces différentes situations de formation.

En second lieu, et de manière très significative, la valeur de la formation est mesurée exclusivement à l'aune de l'augmentation salariale qu'elle est censée servir, tandis que l'augmentation salariale est implicitement interprétée comme le résultat d'une activité préalable de formation. Cette relation réciproque entre formation et salaire s'avère doublement réductrice : d'une part, elle réduit l'impact de la formation à la seule question salariale; d'autre part, elle lie toute augmentation du salaire à l'accroissement des qualifications ou des compétences. Une telle relation bijective s'inscrit dans la ligne des théories du capital humain, dont les paragraphes qui suivent montrent les limites et les présupposés normatifs.

Si la définition rigoureuse du concept de capital humain est absente des travaux de Becker (1964, 1975), depuis Mincer (1974) on retrouve, dans la littérature, de nombreux travaux portant sur l'évaluation 
empirique de la théorie du capital humain, à partir de l'estimation d'une fonction de gains liant les rémunérations à l'accumulation de capital humain. Dans sa version la plus sommaire, cette théorie repose sur deux postulats de base : (1) les inégalités de salaire découlent d'inégalités en capital humain et (2) les inégalités en capital humain proviennent elles-mêmes des comportements individuels (prise d'initiative et de responsabilité)

Or, les limites d'une telle conception, qui est sousjacente à l'indicateur de progrès de qualification, sont nombreuses. Même dans une vision néoclassique du marché du travail, cette conception ne tient compte ni des difficultés pour le salarié à faire reconnaître la vraie valeur de son capital humain ( $c f$. la théorie du signal), ni de l'information imparfaite à laquelle le salarié est soumis, ni des mauvaises anticipations qu'il est susceptible de réaliser.

La formation conçue comme capital humain est au principe même de la matrice de transition de l'indicateur d'évolution de la qualification ( $c f$. tableau 2). Cet ancrage dans la perspective du capital humain se lit également dans une note du « groupe sur les indicateurs » du Comité de l'emploi : «Une modification salariale est interprétée comme une évolution des qualifications de la personne résultant de la formation tout au long de la vie. $»^{4}$ (Commission européenne, 2009b)

Il existe, selon nous, deux manières différentes de concevoir les attendus de la formation : d'une part, comme perspective d'accroissement du capital humain, comme le propose l'indicateur de progrès de qualification, et, d'autre part, comme perspective de développement professionnel, tel que proposé par Zimmermann (2011). Tandis que seul le rendement salarial et promotionnel importe dans le capital humain, le développement professionnel prend en compte les réalisations qu'autorise la formation, en matière d'ouverture des possibles et de potentiel de développement de carrière et de bien-être général (Corteel \& al. 2009 ; Zimmermann, 2011 ; Caillaud \& Zimmermann, 2011 [dans ce numéro]). Le développement professionnel n'est pas forcément synonyme d'évolution de carrière, tout du moins ne s'y limite-t-il

\footnotetext{
4 "A change of pay is interpreted as a change of the person's qualifications as results of lifelong learning".
}

pas. Il désigne une capacité à se former et, au-delà, une capacité à aspirer ${ }^{5}$, une capacité à s'exprimer et se faire entendre, une capacité à exercer un travail de qualité dans un emploi de qualité, ou encore la capacité à équilibrer vie familiale et vie professionnelle (Lambert \& al., 2010).

L'évaluation de la flexicurité se cristallise autour des questions de rendement salarial ou de retour à l'emploi, qui relèvent de la première vision de la formation. Or, les travaux quantitatifs montrent que les effets de la formation continue sont faibles, aussi bien en termes de salaire ou de carrière pour les salariés en place (Fougère, Goux, \& Maurin 2001 ; Dupray \& Hanchane, 2003 : Gadéa \& Trancart, 2003) qu'en termes de retour à l'emploi pour les chômeurs (Marx \& al., 2010). Si la formation est appelée à contribuer à la liberté professionnelle et à la sécurisation des parcours des salariés, elle ne peut à elle seule satisfaire ces objectifs. Il nous semble donc urgent de passer d'une stricte logique comptable d'analyse des effets de la formation en termes de capital humain à une logique qui tienne compte de l'articulation de la formation aux autres facteurs qui pèsent sur le développement de l'agir individuel.

Ces deux conceptions de la formation permettent aussi de préciser la manière dont est envisagée la responsabilité individuelle. Dans la théorie de Becker, tout comme dans l'indicateur de progrès de qualification, les inégalités de capital humain découlent des comportements individuels. Lier la qualification aux seules variables individuelles revient à supposer que la question de la formation ne relève que d'inclinaisons personnelles. En plaçant ainsi l'individu au centre, l'approche en termes de capital humain participe d'un double mouvement qui, d'une part, valorise la singularité individuelle et, d'autre part, enjoint tout un chacun à prendre ses responsabilités et à être le dépositaire de son capital humain. Cette vision fait porter aux travailleurs la responsabilité de l'accès à la formation, indépendamment des contraintes structurelles qui pèsent sur eux. Dès lors, les conditions sont-elles effectivement réunies pour que les travailleurs soient en mesure de développer leurs capacités ? De fait, l'accès à

\footnotetext{
5 Nous définissons la capacité à aspirer comme une capacité à se projeter et à formuler des attentes tournées vers l'avenir (Lambert et al. 2010 ; Lambert et Vero, 2010).
} 
la formation tout au long de la vie n'est pas qu'une question individuelle; il dépend aussi des dispositifs mis en place par les employeurs, partenaires sociaux et institutions publiques. Les travaux conduits dans le cadre du projet Capright montrent ainsi que, au-delà de leur parcours antérieur, c'est l'environnement façonné par l'entreprise qui influence le plus la capacité des salariés à se former. Dans les entreprises qui combinent opportunités de formation pour tous et existence d'espaces de discussion sur la formation, la probabilité que les salariés expriment des aspirations à se former augmente de $60 \%$ (Lambert \& Vero 2010 ; Lambert \& al. 2010). Parmi les facteurs qui influencent la capacité des salariés à se former, Zimmermann (2011) souligne le rôle décisif d'une politique de formation orientée à la fois vers le développement économique de l'entreprise et le développement socioprofessionnel des salariés.

Enfin, l'approche en termes de capital humain, développée au travers des indicateurs de flexicurité, exige de la personne qu'elle serve les objectifs d'adaptabilité, de polyvalence et de responsabilité. Elle vise à accroître le rendement du capital humain en mettant la personne au service d'objectifs d'efficacité économique et de performance. Le modèle de flexicurité, tel qu'il se traduit dans l'indicateur de progrès de qualification, propose une stratégie de la sécurité par l'employabilité dont l'ambition est de faciliter la prise d'un nouvel emploi au sein de la même entreprise ou chez un autre employeur. Il répond ainsi à l'impératif économique de réactivité et de flexibilité, mais néglige la prise en compte de la dynamique temporelle et sociale des parcours individuels, des préférences et des choix personnels.

Or, dans l'approche par les capacités, l'épanouissement des êtres humains n'est pas seulement un moyen pour augmenter la prospérité nationale ou européenne (ce qui est sous-jacent à la conception du capital humain), mais aussi la finalité en soi de toutes les politiques économiques ou sociales. Tout en reconnaissant la nécessité de s'adapter aux changements de la production et aux nouvelles compétences des emplois, la perspective des capacités se démarque de l'employabilité conçue comme stricte adaptabilité aux exigences du marché du travail. Elle requiert d'octroyer aux personnes des opportunités leur permettant d'acquérir les moyens de la liberté de choix. Elle nécessité également le respect de la liberté processuelle grâce à laquelle les personnes restent maîtresses de leurs choix. Que l'une ou l'autre de ces deux dimensions fasse défaut et l'objectif de développement des capacités n'est pas atteint. Une telle perspective suppose l'accès à l'information et la possibilité de pouvoir débattre, proposer, contester et s'opposer à des orientations que l'on désapprouve, autant de considérations absentes des indicateurs de flexicurité.

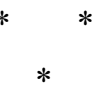

Dans un contexte de gouvernance inspiré par la nouvelle gestion publique, celui de la Méthode ouverte de coordination, la mesure par les indicateurs de performance ne se résume pas à une question purement technique ; elle comporte également des injonctions fortes en termes d'objectifs à atteindre. Dès lors, au travers de ce que l'on veut mesurer, il convient de se demander quelle vision de la flexicurité on souhaite promouvoir. Les directives européennes visent avant tout le développement de l'employabilité conçue comme adaptabilité accrue aux exigences du marché du travail. Elles visent à instaurer de nouvelles formes de sécurité par l'employabilité. Il s'agit de faciliter la prise d'un nouvel emploi au sein de la même entreprise ou chez un autre employeur. Cette vision de la sécurité par l'employabilité ne résiste pas à l'approche par les capacités, qui suggère une autre conception de la flexicurité et donc d'autres instruments et indicateurs pour la mesurer.

En conclusion, nous souhaitons plaider, dans la ligne des travaux réalisés dans les réseaux Eurocap et Capright ${ }^{6}$, en faveur d'une conception renouvelée des bases de données susceptible de promouvoir cette autre conception de la flexicurité. Dans un premier temps, nous analyserons la contribution normative de l'approche par les capacités, qui vise à mettre en discussion les valeurs sous-jacentes aux indicateurs mis en place par l'EMCO, afin d'élargir le débat sur l'employabilité à la question des capacités. Dans un

${ }^{6}$ Eurocap (2002-2006) et Capright (2007-2010) sont deux projets européens de recherche relevant respectivement du $5^{\mathrm{e}}$ et $\mathrm{du}$ $6^{\text {e }}$ Programme Cadre de la DG Recherche de l'Union européenne. Coordonnés par Robert Salais, ils visent à relancer l'Europe sociale sur de nouvelles bases, en appliquant l'approche par les capacités introduite par A. Sen dans les domaines de l'emploi, du travail, de la formation, et de la protection sociale. 
second temps, nous dessinerons les objectifs que vise l'AC pour améliorer et compléter les sources statistiques existantes.

\section{DOCUMENTER LES DIMENSIONS CONSTITUTIVES DU DÉVELOPPEMENT PROFESSIONNEL}

Aujourd'hui, les indicateurs dynamiques révèlent un certain nombre de faiblesses. D'une part, ils engagent dans une documentation de la flexicurité qui privilégie la flexibilité du marché du travail. Ils attendent des travailleurs qu'ils s'adaptent aux exigences du marché du travail, tout occultant la question de la liberté réelle des personnes concernées (dans le sens de la liberté processuelle). En étroite corrélation avec cela, la seconde faiblesse de ces indicateurs est qu'ils indexent la sécurisation des parcours, conçue comme adaptabilité unilatérale aux exigences du marché du travail, à la seule responsabilité individuelle.

Par contraste, dans la perspective des capacités, la question n'est pas de savoir si les travailleurs sont plus flexibles ou adaptables. Il s'agit plutôt de savoir si les conditions sont effectivement réunies pour que les salariés disposent d'une liberté réelle de travailler et de se développer professionnellement ; cette liberté constituant une condition préalable pour prendre une part active dans la transition externe d'un emploi à l'autre ou dans la transition interne à une entreprise visant un changement de poste, de service ou de métier. Les recherches empiriques montrent que la sécurisation des parcours progresse, d'une part, quand les pouvoirs publics soutiennent les salariés et les entreprises en leur offrant un cadre institutionnel leur permettant la prise de risque et de responsabilité (Keune \& Pochet, 2009); d'autre part, quand les entreprises développent des « organisations capacitantes » qui orientent la formation à la fois vers le développement de l'entreprise et le développement professionnel des salariés (Zimmermann, 2011).

Les travaux conduits à l'occasion des recherches Eurocap et Capright ont permis d'identifier quelques capacités qui contribuent au développement professionnel dans l'entreprise. Celui-ci engage la capacité à se former, mais au-delà de la formation, comptent aussi la capacité à exprimer son point de vue et le faire entendre, la capacité à aspirer et à se projeter vers l'avenir, mais aussi la capacité à réaliser un travail de qualité dans un emploi de qualité ou encore la capacité à équilibrer vie familiale et vie professionnelle (Zimmermann, 2011 ; Lambert \& al., 2010). Ces capacités s'apprécient dans la dynamique des parcours et les interactions avec l'environnement professionnel : «En tant que processus, [le développement professionnel] se déploie dans le temps et amène à explorer la dynamique temporelle des parcours (...) En tant qu'interaction sociale, il engage la relation d'une personne à son environnement » (Lambert \& al., ibid, p. 12).

Une telle conception implique donc une refonte des bases informationnelles de jugement, au fondement des enquêtes, dans le sens d'une meilleure prise en compte de la dimension temporelle et des interactions $\mathrm{du}$ travailleur avec son environnement, deux dimensions quasi absentes dans la conception des indicateurs dynamiques de flexicurité.

\section{Renforcer la dimension longitudinale}

Afin d'explorer le processus de développement des personnes, nous plaidons, avec Orianne et al. (2010), pour un renforcement de la perspective longitudinale des dispositifs d'observation. Les matrices de transition à l'origine des indicateurs dynamiques de flexicurité présentent l'inconvénient majeur de faire l'impasse sur le déroulement du parcours entre le point de départ (année T) et le point d'arrivée (année $\mathrm{T}+1$ ). Tout se passe comme si, entre ces deux dates, les événements qui se produisent n'ont pas d'incidence sur la compréhension du cheminement du salarié. Ce faisant, les indicateurs n'accordent que peu de place à la prise en compte de la dynamique temporelle. Or, il nous paraît primordial de prendre en compte l'intégralité du processus et de ne pas se centrer sur les seules transitions annuelles.

\section{GÉNÉRALISER LA COLLECTE D'ENQUÊTES COUPLÉES}

Le développement professionnel doit aussi s'envisager dans les interactions du travailleur avec son 
environnement. L'extension de la gamme de choix de formation des personnes, de leur capacité à aspirer, à participer aux décisions qui les concernent, à exercer un travail de qualité dans un emploi de qualité, tout comme la possibilité de concilier de manière harmonieuse la sphère privée et celle du travail supposent de pouvoir opérer des choix en lien avec l'environnement.

À cet égard, les organisations économiques jouent un rôle central. Le caractère décisif de l'environnement offert par l'entreprise, tant sous l'angle de la participation que de l'accès à la formation ou aux autres dimensions du développement professionnel, plaide pour un dispositif d'observation associant plusieurs niveaux d'enquêtes auprès des personnes et des organisations au sein desquelles elles évoluent. Lambert et $a l$. (2010), grâce à l'analyse des caractéristiques des entreprises concourant au développement professionnel de leurs salariés, ont mis au jour les caractéristiques des « organisations capacitantes ». Il en ressort que les politiques des ressources humaines, mais aussi la stratégie d'entreprise, les pratiques de management et l'organisation du travail exercent un rôle majeur qu'il convient d'observer pour comprendre la latitude de choix offerte aux personnes. L'objectif devient donc de construire un système d'information qui permette de corréler les politiques des entreprises avec les parcours des personnes.

La disponibilité des données est cruciale pour promouvoir une autre conception de la flexicurité visant le développement professionnel et l'accroissement des capacités des personnes au travail. Il est en effet essentiel de rassembler le matériau nécessaire pour passer d'une production de la connaissance orientée vers la sécurité par l'employabilité à une sécurité par la liberté de travailler et de se développer professionnellement. Nous défendons ici l'idée que cette documentation passe par l'élaboration de dispositifs d'enquêtes couplées longitudinales, permettant de mettre en rapport les parcours des salariés avec les politiques conduites au sein des entreprises dans lesquelles ils sont employés.

Pour ambitieuses qu'elles paraissent, ces propositions ne sont pas irréalistes. L'Europe dispose déjà de deux grandes enquêtes européennes sur la formation, l'une auprès des individus (FC 2006 appelée encore AES pour Adult Education Survey), l'autre auprès des entreprises (l'enquête CVTS pour Continuing Vocational Training Survey). Un réagencement de celles-ci dans une perspective de couplage, à l'image du dispositif français DIFES1 ${ }^{7}$ (pour Dispositif d'information sur la formation employeur-salarié), permettrait de significatives avancées. La compréhension fine de la dynamique des parcours et des interactions avec l'environnement suppose de se tourner, en outre, vers d'autres démarches plus qualitatives qui, n'ayant pas d'objectif de représentativité, peuvent permettre de progresser dans la compréhension des mécanismes de sécurisation des parcours. Mais l'enjeu consiste à savoir si l'on souhaite, à l'occasion du débat sur la flexicurité, revoir les instruments de mesure de la flexicurité, ce qui supposerait en amont une autre manière de concevoir les objectifs des politiques du marché du travail.

\footnotetext{
${ }^{7}$ Le Dispositif d'information sur la formation employeursalarié (DIFES1) croise, pour la première fois en France, les réponses de 1800 salariés avec celles de leurs employeurs sur le thème de la formation continue dans l'entreprise. Il est construit à partir des deux enquêtes FC2006 et CVTS3. Le dispositif DIFES1 permet de lier les caractéristiques des salariés et leurs pratiques de formation à la stratégie économique de l'entreprise, aux changements organisationnels, à sa politique de gestion des ressources humaines et de formation. Pour une présentation du dispositif DIFES1, se rapporter au site du céreq : http://www.cereq. fr/EnqueteDIFE1.htm
}

\section{Bibliographie}

Becker G. S. (1964), Human Capital: A Theoretical and Empirical Analysis, with Special Reference to Education. Chicago, University of Chicago Press.
Becker G. S. (1975), Human Capital: A Theoretical and Empirical Analysis, with Special Reference to Education. New York, Columbia University Press. 
Bonvin J.-M., Conter B. (2006), Les politiques locales de l'emploi, reflet des nouvelles logiques d'interventions publiques, Colloque État et Régulation Sociale, organisé par le Matisse-Cnrs, 11-13 sept, Paris.

Bonvin J.-M. (2005), « La démocratie dans l'approche d'Amartya Sen », L'Économie politique $3 / 2005, n^{\circ} 27$, pp. 24-37.

Bonvin J.-M., Vielle P. (2009), "Une flexicurité au service des capacités des citoyens européens ", La Revue de l'Ires. Vol. 63, n 4, pp. 17-33.

Caillaud P., Zimmermann B. (2011), «Sécurisation des parcours et liberté professionnelle : De la "flexicurité" aux capacités », Formation Emploi, n 113, mars.

Commission européenne (2001), Employment and Social Policies: a Framework for Investing in Quality, Communication de la Commission du conseil, Bruxelles.

Commission européenne (2006a), Rapport sur l'emploi en Europe 2006, OPCE, Bruxelles.

Commission européenne (2006b), Livre vert. Moderniser le droit du travail pour relever les défis $d u$ XXI siècle, COM (2006) 708 final, 22 novembre.

Commission européenne (2007), Vers des principes communs de flexicurité : des emplois plus nombreux et de meilleure qualité en combinant flexibilité et sécurité, Bruxelles, [COM(2007) 359 final].

Commission européenne (2009), Monitoring and Analysis of Flexicurity Policies, EMCO Report, Issue 2, July.

Corteel D., Lambert M., Vero J., Zimmermann B. (2009), "Capability for learning in French companies", Net.doc, n 50, pp. 115-150, http://www.cereq. fr/pdf/Net-Doc-50.pdf

Desrosières A. (2008), L'argument statistique I Pour une sociologie historique de la quantification, Paris, Presses de l'École des Mines.

Dupray A., Hanchane S. (2003), «La formation continue en entreprise et ses effets sur le salaire et la mobilité des jeunes ", in Lochet J.-F. Entreprises et jeunes débutants Paris, Céreq et L'Harmattan.

Employment Committee (2009), Mobility - Proposal for interpretation of transition indicators and presentation in output radar charts, INDIC/12/300309/EN.

Esping-Andersen G. (1999), Social Foundations of Postindustrial Economies, Oxford, Oxford University Press.

Esping-Andersen G. (2007), Les trois mondes de l'État providence, Essai sur le capitalisme moderne (traduction révisée), Paris, PUF.

Fougère D., Goux D., Maurin E. (2001), « Formation continue et carrières salariales : une évaluation sur données individuelles ", Annales d'économie et de statistique, $\mathrm{n}^{\circ} 62$, pp. 49-69.

Gadéa C., Trancart D. (2003), « Pratiques de formation continue et promotion au statut de cadre : un lien paradoxal », Formation Emploi n ${ }^{\circ}$ 81, pp. 99-113.

Gadrey J., Jany-Catrice F. (2005), Les nouveaux indicateurs de richesse, Paris, La Découverte.

Gazier B., Lechevalier A. (2006), "Stratégie Européenne de l'Emploi, régimes macroéconomiques et institutionnels, et Marchés Transitionnels du Travail », in Dang A.-T., Outin J.-L., Zajdela H. (eds.), Travailler pour être intégré? Mutation des relations entre emploi et protection sociale, Paris, CNRS- Éditions, pp. 218-234.

Hall P.A., Soskice D. (eds) (2001), Varieties of Capitalism: The institutional Foundations of Comparative Advantage, Princeton and Oxford, Princeton University Press.

Keune M., Pochet P. (2009), « Flexicurité en Europe: une approche critique ", La revue de l'IRES, $\mathrm{n}^{\circ}$ 63, pp. 105-126.

Lambert M. et Vero J. (2010), «Aspirer à se former. La responsabilité des entreprises en question », Bref, $\mathrm{n}^{\circ} 279$, novembre.

Lambert M., Vero J., Subrumanian D. et Zimmermann B. (2010), «Formation et Développement profes- 
sionnel. La responsabilité des entreprises en question », Communication présentée lors de la conférence finale du projet Capright, Nantes, 2 et 3 décembre. http://www.capright.eu/

Marx J.-M. (Rapport du groupe de travail présidé par) (2010), La formation professionnelle des demandeurs d'emploi, Paris, Secrétariat d'État à l'Emploi.

Méda D. (2008), Au-delà du PIB : Pour une autre mesure de la richesse, Paris, Flammarion.

Mincer J. (1974), Schooling, Experience, and Earnings, New York, Columbia University Press for NBER (National Bureau of Economic Research).

Muffels R., Luijkx R. (2008), "Labour market mobility and employment security of male employees in Europe: 'trade-off' or 'flexicurity'", Work Employment Society, vol. 22, n 2, pp. 221-242.

Orianne J.-F., Pichault F., Levêque A. (2010), Le développement d'un cadre d'indicateurs relatifs à des transitions positives et à la flexicurité dans le contexte des relations individuelles du travail, Rapport réalisé pour le SPF Emploi, Travail et Concertation sociale, université de Liège.

Perret B. (2002), Indicateurs sociaux, état des lieux et perspectives, Rapport au Conseil de l'Emploi, des Revenus et de la Cohésion Sociale (CERC).

Raveaud G. (2006), « La Stratégie européenne pour l'emploi : une politique d'offre de travail », Travail et Emploi, $\mathrm{n}^{\circ} 107$, pp. 7-18.

Salais R. (2004), « La politique des indicateurs. Du taux de chômage au taux d'emploi dans la Stratégie Européenne pour l'Emploi » in B. Zimmermann (dir.), Les sciences sociales à l'épreuve de l'action: le savant, le politique et l'Europe, Paris, Éditions de la MSH, pp. 287-331.

Salais R. (2005), « Le projet européen à l'aune des travaux de Sen », L'Économie politique, 3/2005, $n^{\circ} 27$, pp. 8-23.

Salais R. (2010), «Usages et mésusages de l'argument statistique : le pilotage des politiques publiques par la performance ", Revue Française des Affaires Sociales, $\mathrm{n}^{\circ} 1-2$, pp. 129-147.

Schmid G., Gazier B. (eds) (2002), The dynamics of full employment, Social integration through transitional labour markets, Cheltenham, Edward Elgar.

Sen A (1993), « Positional objectivity », Philosophy and Public Affairs, $\mathrm{n}^{\circ} 22$ (2), pp. 83-135, reproduit dans Sen A. (2005), Rationalité et liberté en économie, Paris, Odile Jacob.

Sen A. (2010), L'idée de justice, Paris, Flammarion.

Stiglitz J., Sen A. et Fitoussi J.-P. (2009), Vers de nouveaux systèmes de mesure, Paris, Odile Jacob.

Supiot A. (2010), L'esprit de Philadelphie, la justice sociale face au marché total, Paris, Seuil.

Supiot A. (dir.) (1999), Au-delà de l'emploi. Transformations $d u$ travail et devenir $d u$ droit $d u$ travail en Europe, Paris, Flammarion.

Wilthagen T. (1998), "Flexicurity: A New Paradigm for Labour Market Policy Reform?”, Flexicurity Research Programme, FXPaper Nr. 1.

Wilthagen T., Tros F. (2004), “The concept of flexicurity: a new approach to regulating employment and labour markets", Transfer, vol. 10, n 2, pp. 166-186.

Wilthagen T., Tros F., van Lieshout H. (2003), Towards "flexicurity"? Balancing flexibility and security in EU member states, Document présenté lors du $13^{\mathrm{e}}$ Congrès mondial de l'Association international des relations collectives de travail. Berlin, septembre.

Zeitlin J., Pochet P. (eds) (2005), The Open Method of Co-ordination in Action: The European Employment and Social Inclusion Strategies, Bruxelles, Peter Lang.

Zimmermann B. (2011), Ce que travailler veut dire. Une sociologie des capacités et des parcours professionnels, Paris, Economica, Collection «Études sociologiques $»$. 


\title{
Résumé
}

\section{Déchiffrer deux indicateurs européens de flexicurité à l'aune de l'approche par les capacités}

\author{
Jean-Michel Bonvin, Éric Moachon et Josiane Vero
}

Depuis 2007, la question de l'équilibre entre flexibilité et sécurité sur le marché du travail est devenue une des priorités de l'agenda de l'Union européenne. Les efforts des États-Membres en la matière sont notamment évalués au moyen des deux seuls indicateurs dynamiques qui mesurent la sécurité de l'emploi et le progrès des qualifications. Cet article vise à clarifier les fondements normatifs de ces instruments de mesure. Se basant sur l'approche par les capacités d'Amartya Sen, il discute les limites de ces fondements normatifs qui privilégient flexibilité plutôt que liberté réelle des travailleurs, employabilité plutôt que capacité et capital humain plutôt que développement professionnel. II propose une conception alternative de la sécurité de l'emploi et du rôle qu'est susceptible d'y jouer la formation. II conclut en présentant quelques repères afin d'élaborer des indicateurs davantage inspirés par l'approche par les capacités.

Mots clés

Approche par les capabilités, sécurisation des parcours professionnels, MOC (méthode ouverte de coordination) Union européenne, évaluation, formation tout au long de la vie

Journal of Economic Literature : J 44, D 78 